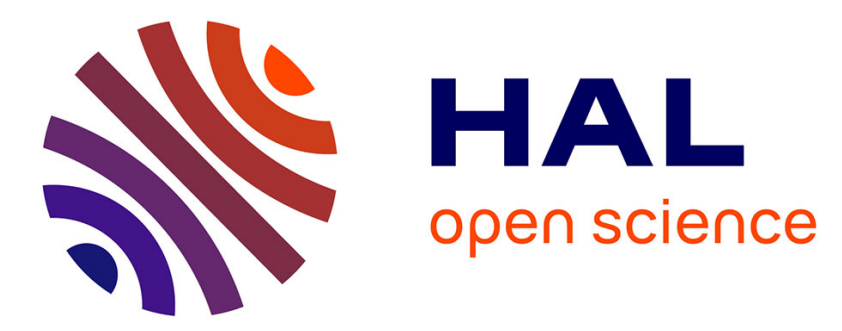

\title{
Monolayer Structures of 4,4' Bipyridine on Graphite at Sub-monolayer Coverage
}

Stuart Matthew Clarke, Tomislav Friščić, Anasuya Mandal, Chenguang Sun, Julia E Parker

\section{- To cite this version:}

Stuart Matthew Clarke, Tomislav Friščić, Anasuya Mandal, Chenguang Sun, Julia E Parker. Monolayer Structures of 4,4' Bipyridine on Graphite at Sub-monolayer Coverage. Molecular Physics, 2011, 109 (03), pp.477-481. 10.1080/00268976.2010.531296 . hal-00669190

\section{HAL Id: hal-00669190 \\ https://hal.science/hal-00669190}

Submitted on 12 Feb 2012

HAL is a multi-disciplinary open access archive for the deposit and dissemination of scientific research documents, whether they are published or not. The documents may come from teaching and research institutions in France or abroad, or from public or private research centers.
L'archive ouverte pluridisciplinaire HAL, est destinée au dépôt et à la diffusion de documents scientifiques de niveau recherche, publiés ou non, émanant des établissements d'enseignement et de recherche français ou étrangers, des laboratoires publics ou privés. 


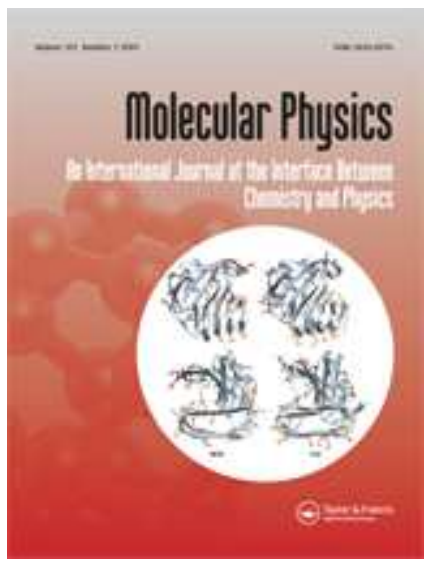

\section{Monolayer Structures of 4,4' Bipyridine on Graphite at Sub-monolayer Coverage}

\begin{tabular}{|r|l|}
\hline Journal: & Molecular Physics \\
\hline Manuscript ID: & TMPH-2010-0339.R1 \\
\hline Manuscript Type: & Full Paper \\
\hline Author: & $30-$ Sep-2010 \\
\hline Complete List of Authors: & $\begin{array}{l}\text { Clarke, Stuart; University of Cambridge, Chemistry } \\
\text { Frišćć, Tomislav; University of Cambridge } \\
\text { mandal, Anasuya; Indian Institute of Technology } \\
\text { Sun, Chenguang; University of Cambridge } \\
\text { parker, julia; DIAMOND Light source }\end{array}$ \\
\hline Keywords: & monolayers, Physisorption, synchrotron \\
\hline \multicolumn{2}{|l}{} \\
\hline
\end{tabular}

\section{SCHOLARONE Manuscripts}




\title{
Monolayer Structures of 4,4' Bipyridine on Graphite
}

\author{
at Sub-monolayer Coverage \\ S.M. Clarke*, T. Friščić, A. Mandal and C. Sun \\ BP Institute and Department of Chemistry, University of Cambridge, Cambridge, UK
}

\author{
J. E. Parker \\ Diamond Light Source, Diamond House, Harwell Science and Innovation Campus, Didcot, \\ Oxon. \\ *Address correspondence to this author: stuart@bpi.cam.ac.uk
}

\begin{abstract}
The formation of crystalline monolayers of 4,4'bipyridine at submonolayer coverage have been characterised by synchrotron X-ray diffraction. The experimentally determined monolayer unit cell exhibits a small temperature dependence, such that the monolayer structure at higher temperatures has a square unit cell, while at lower temperatures the unit cell becomes very slightly rectangular. Both structures have the same plane group, Pgg. The experimentally determined lattice parameters are $a=11.42 \AA, \mathrm{i}=11.42 \AA$ and $v\left(=90^{\circ}\right.$ and $a=11.26 \AA, b=11.45 \AA, v=90^{\circ}$ for the high and low temperature structures, respectively. There are two molecules per unit cell with the pyridine units of each molecule in a plane parallel to the graphite substrate, although we are not particularly sensitive to this orientation. Interestingly, the monolayer does not melt even at the highest temperatures accessible on the device, $445 \mathrm{~K}$, well above the bulk melting point. This is a very unusual behaviour particularly at these submonolayer coverages.

Keywords: Monolayers, Synchrotron, Physisorption
\end{abstract}




\section{Introduction}

The structure and properties of physisorbed monolayers is of continuing interest. In particular, the influence of non-covalent interactions within the monolayer, such as hydrogen bonding, have been found to lead to the formation of very stable solid monolayers ${ }^{1-4}$ even at temperatures above the bulk melting point where the bulk adsorbate is liquid. Usually such solid monolayers can form at high, multilayer coverages. However, recent evidence has indicated that alkyl amides can form stable solid monolayers at temperatures well above the bulk melting point, even at submonolayer coverages ${ }^{1}$.

In this work we report the formation of solid monolayers of 4,4 'bipyridine at submonolayer coverages on the surface of graphite. This and closely related molecular species are important in a variety of areas, but most particularly as ligands used to complex with other species in the area of molecular engineering and surface self assembly. ${ }^{5-7}$ Here we endeavour to understand the structure and behaviour of the monolayer of the pure material. In addition, the 4,4'-bipyridine molecule has the potential for a number of interactions on its own arising from the heterocyclic nature of the species and the aromatic nature of the bonding. In particular, the molecule can self-assemble through the formation of weak hydrogen bonds ${ }^{8,9}$ of the N... H-C or C$\mathrm{H} \ldots \pi$ type. In this work we report the sub monolayer structures of the 4,4 ' bipyridine molecule on graphite determined by synchrotron X-ray diffraction.

\section{Experimental}

The procedures for synchrotron X-ray experiments have been described elsewhere ${ }^{1}$. The instrument used was $111^{10}$ at the Diamond Light Source, Didcot, UK with a wavelength $1.062437 \AA$, determined with a silicon standard (NIST SRM 640c). The zero angle off-set was similarly determined to be $-0.0117^{\circ}$. The adsorbent used was recompressed exfoliated graphite, Papyex, from Le Carbon, with a specific surface area of $28 \mathrm{~m}^{2} / \mathrm{g}$ determined by nitrogen adsorption. This material is essentially a powder, but with a significant extent of preferred orientation that was exploited in the experiments to optimise the collection of monolayer scattering. The 4,4 ' bipyridine ${ }^{4}$ (BPY) was obtained from Sigma with a purity of 99.3\%, as stated on the certificate of analysis, and was used without further purification.

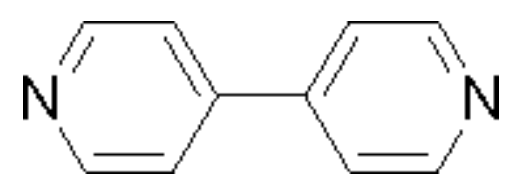

Figure 1. The molecular structure of 4,4' bipyridine (BPY)

The experiments employed a single glass capillary with $2 \mathrm{~mm}$ diameter discs of graphite stacked so that the scattering vector of the radiation was in the plane of the surface of the preferentially oriented crystallites. Approximately $4 \mathrm{~g}$ of graphite was dosed by adding the appropriate amount of the BPY to give a coverage of 0.5 


\section{Results}

Figure 2 presents the synchrotron X-ray diffraction data from BPY at 0.5 monolayer coverage. Figure 2(a) indicates the temperature dependence of the pattern. Figure 2(b) gives a typical pattern at higher temperatures (423K) and Figure 2(c) at the lower temperature of $260 \mathrm{~K}$. The bare graphite diffraction pattern has been subtracted in preparing this figure and hence the peaks are those from the adsorbed layer. The strong small angle, 'Porod' scattering from the graphite crystallites was also partly removed. The strong peaks in the diffraction pattern appear at $2 \theta$ approximately $7.6^{\circ}$ and $12.0^{\circ}$, with the characteristic 'saw-tooth' lineshape characteristic of twodimensional (2D) layers, ${ }^{11} 12$ clearly indicating that BPY does form a solid monolayer under these conditions. The sharp 'spike' feature at approximately $5.7^{\circ}$ arises from a single detector element inefficiency.

On raising the temperature it was found that the monolayer did not melt even at the highest temperature (445K) accessible with the present experimental configuration. The bulk melting point of BPY is $110^{\circ} \mathrm{C}(383 \mathrm{~K})$. This represents an elevation of at least $16 \%$ above the bulk melting point. The single high angle peak at high temperatures is seen to be split into two peaks at low temperatures. This suggests some structural modification, such as a lowering of the monolayer symmetry, on cooling.

We have performed differential scanning calorimetry on this sample and have observed a transition at a temperature of approximately $169^{\circ} \mathrm{C}(442 \mathrm{~K})$ in both heating and cooling. There are no transitions for the bare graphite in this temperature region so it is most likely to be the monolayer melting point. Thus we conclude that the diffraction data was collected over a temperature range that ended just at the monolayer melting point. The reduction in symmetry of the diffraction pattern that is observed as the melting point is approached, is similar to several alkyl species, such as alkanes, where higher symmetry 'rotator' phases are formed just prior to melting ${ }^{13}$ in the pure alkanes and their mixtures. Hence we conclude that the monolayer melting point is approximately $442 \mathrm{~K}$.

The diffraction data were analysed as described previously. ${ }^{2}$ Because there are very few diffraction peaks it is important to constrain the fitting procedure as much as possible. Here we have taken the molecular structure unchanged from the bulk ${ }^{14}$ 
although we consider that the two pyridine rings are coplanar without any twist that may arise from interactions between hydrogen atoms which are rather close. As discussed below, our measurements are not sensitive to this particular aspect of molecular structure. In addition, we assess high symmetry plane groups with few degrees of freedom before assessing those with lower symmetry and hence more independent variables. The two dimensional lineshape of Warren and Kjiems ${ }^{11}{ }^{12}$ has been used. This model includes the preferred orientation of the substrate and the $2 \mathrm{D}$ crystallite size. The resolution function of the I11 instrument was approximated with a Gaussian convolution. A single temperature factor was set to unity in this simple model.

It is found that the data in Figure 2(b) are well fitted by a square unit cell with lattice parameters, $a=11.42(2) \AA, b=11.42(2) \AA$ and $\mathrm{v}=90.0(2)^{\circ}$. This unit cell has two molecules with an overall plane group of $p g g$, as schematically illustrated in Figure 3(a). The carbon atoms all lie in a plane parallel to the graphite surface. In fitting the orientation of the molecules in this structure the model is highly constrained. If we consider that the molecules adopt the most symmetric orientation with respect to the unit cell, aligned diagonally at $45^{\circ}$ to the a-axis, then there are no degrees of freedom in fitting this pattern. This results in the fit shown in Figure 2(b), which is seen to be in very good agreement with the experimental pattern. This fit can be refined if we vary the orientational angle of the molecules with respect to the $a$-axis of the cell. However, within the experimental resolution, $+/-5^{\circ}$, this angle remains $45^{\circ}$. Table 1 gives the symmetry related positions of the Pgg plane group.

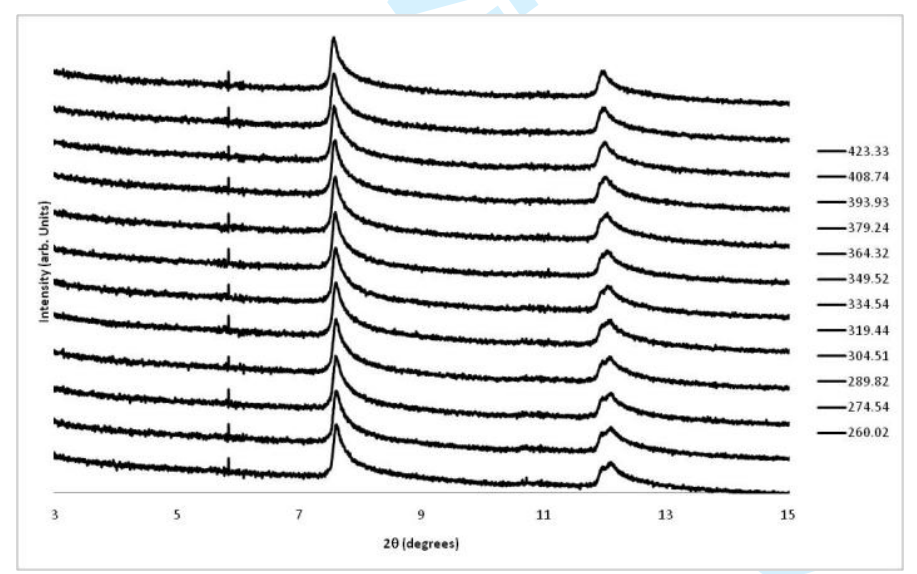

(a) 


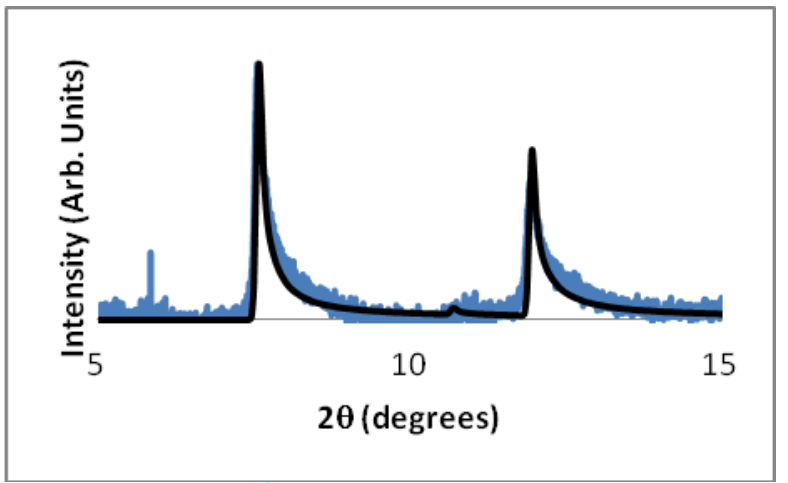

(b)

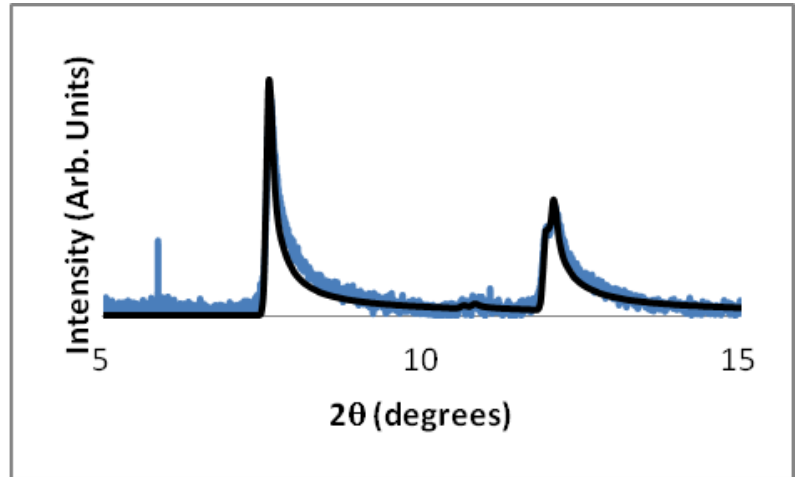

(c)

Figure 2. Diffraction data from 0.5 monolayers of BPY on graphite. (a) The temperature dependence of the experimental scattering patterns. (b) The experimental and calculated diffraction patterns at $423 \mathrm{~K}$, high temperature. (c) Experimental and calculated diffraction patterns at $260 \mathrm{~K}$, low temperature.

Rotation of the molecule about its long molecule axis has very little effect on the calculated pattern and hence there is significant uncertainty if the molecules are flat or not. However, the structure proposed in Figure 3 also shows excellent close packing arrangement of the molecules when they are flat on the surface that the upright arrangement does not give.

The 'goodness of fit', R parameter, has been calculated using Equation 1:

$R=\frac{\Sigma_{H, K}\left(U_{o b s}-I_{c a l c}\right)^{2}}{\Sigma_{H, K}\left(U_{o b s}\right)^{2}}$ (Equation 1)

Typical $\mathrm{R}$ values are found to be in the range of 0.003 to 0.011 for the fits in Figure 2 (b) and (c). However, these are only over 20 reflections in the accessible $q$-range of which most have very small or zero intensity, and so cannot be readily compared with conventional three-dimensional X-ray crystallography.

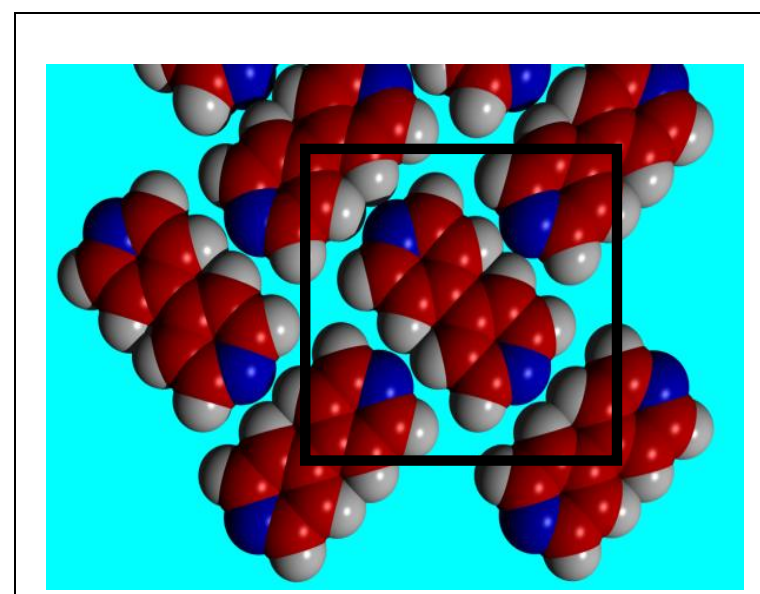

(a)

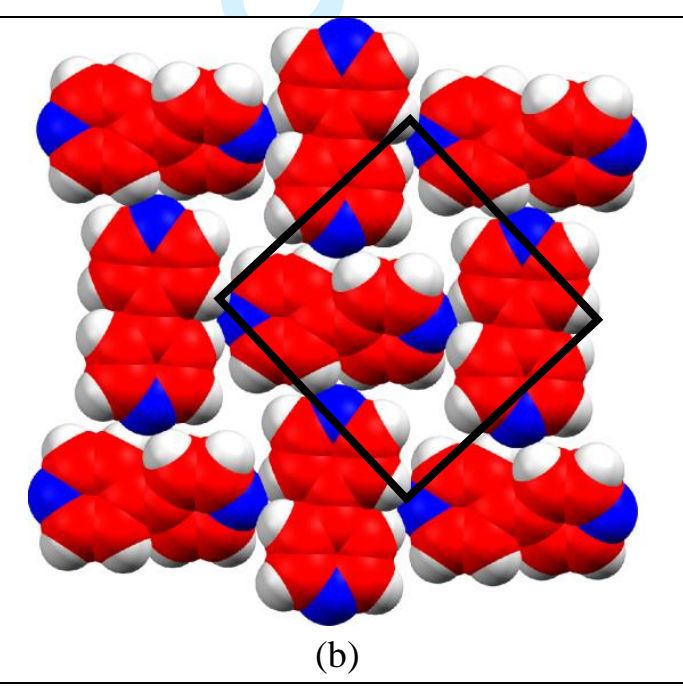


Figure 3.(a) Experimentally determined monolayer structure of BPY high temperature phase with a square unit cell and pgg plane group. The solid line indicates the unit cell. The structure of the slightly oblique unit cell of the low temperature phase is essentially indistinguishable. Key: Blue = nitrogen, Black $=$ red, Grey $=$ hydrogen (b) Bulk [110] plane. The unit cell is also indicated.

It is found that the data in Figure 2(c) are well fitted by a slightly rectangular unit cell with lattice parameters, $a=11.26(2) \AA, b=11.45(2) \AA$ and $v=90.0(2)^{\circ}$, which is slightly compressed relative to the higher temperature phase discussed above. A compression on lowering the temperature is to be expected. This unit cell also has two molecules with an overall plane group of pgg. If we consider that the molecules remain flat on the surface and that the second molecule is retained in the centre of the cell, this is a highly constrained fitting with only the orientational angle of the molecule with respect to the $a$-axis of the cell as a fitting variable. The resulting structure is essentially the same as for the high temperature phase, and fitting indicates that this angle is still $45(5)^{\circ}$. The position of the second molecule in the cell can also be refined. However, there is little possible variation in the position of this molecule that is physically reasonable. The solid line through the data in Figure 2(c) represents the calculated diffraction pattern from this structure. The schematic illustration of the structure is essentially indistinguishable from that of the square pgg structure above.

This characteristic splitting of the peaks at $2 \theta=12^{\circ}$ means that the resolution of the experiment is high and that the $2 \mathrm{D}$ monolayer crystallite size must also be relatively high. Without this larger crystallite size the peaks would be too broad to observe the splitting. The fitting indicates that this crystallite size is approximately $650 \AA$.

$$
\begin{array}{cc}
X, Y & -X,-Y \\
1 / 2+X, 1 / 2-Y & 1 / 2-X, 1 / 2+Y
\end{array}
$$

Table 1 . Symmetry related positions under the $P g g$ plane group.

\section{Discussion}

The experimentally determined monolayer structure of BPY illustrated in Figure 3 show that the molecules adopt a zig-zag 'herringbone' molecular arrangement. There is no evidence that the nitrogen atoms on adjacent molecules interact given that they do not face each other but rather face the middle of the two phenyl rings. It is proposed that this orientation facilitates the formation of weak hydrogen bonds between the pyridine $\mathrm{N}$ atoms and the $\mathrm{C}-\mathrm{H}$ groups of the aromatic ring of the neighbouring molecules. The $\mathrm{N}$ to $\mathrm{C}$ distances are determined to be $3.61 \AA$ and $3.66 \AA$ for the low temperature phase and $3.69 \AA$ for the high temperature phase. However, the precise length of these non-covalent bonds must be taken with some caution here. Given the limited number of X-ray reflections we cannot refine every bond length and angle in the molecules as would usually be the case in three-dimensional 
crystallography. Here we have assumed the same molecular structure as the bulk and hence we are only sensitive to variations in intermolecular separation.

This 2D structure solution very closely resembles the structure of the [110] layer in the three-dimensional crystal structure of BPY (Cambridge Crystallographic Database Centre reference codes: HIQWEJ, HIQWEJ01 and HIQWEJ02, Figure 3b). ${ }^{12}$ In the bulk crystal structure, the molecules are interconnected through three-centered hydrogen bonds with the pyridine nitrogen atom as the bifurcated acceptor and $\mathrm{C} \cdots \mathrm{N}$ distances of $3.50 \AA$ and $3.60 \AA$.

It is somewhat surprising that the $\mathrm{C}-\mathrm{H} \cdots \mathrm{N}$ hydrogen-bonding interactions, with a typical strength of ca. $5 \mathrm{~kJ} \mathrm{~mol}^{-1}$ each $^{15}$, , could elevate the sub-monolayer melting point so much above the usual bulk value. A possible explanation would be that the monolayer structure is stabilised by the bifurcated nature of the bond, as well as the rigidity and polyfunctional nature of the BPY molecules: each molecule contains a single torsional angle and acts as a two-fold bifurcated hydrogen bond donor and a two-fold bifurcated hydrogen bond acceptor.

The proposed molecular arrangement also can accommodate effective close packing of the molecules. The possible close packing arrangements in $2 \mathrm{D}$ for a given molecular symmetry has been addressed by Kitaigorodskii ${ }^{16}$ and by Clarke. ${ }^{17}$ The BPY molecule has two symmetry lines and a centre of inversion (equivalent to a $\mathrm{C}_{2}$ axis in 2D). Hence there are several possible close packing plane groups including $\mathrm{Cmm}, \mathrm{Pgg}$ and P2. Here we have presented evidence that the molecules adopt Pgg plane group, consistent with this prediction.

From a simple comparison of the lattice parameters of the overlayer with those characteristic of the graphite surface $\left(a_{\mathrm{g}}=2.46 \AA\right.$ and $\left.3 \sqrt{ } a_{\mathrm{g}}=4.26 \AA\right)$, we cannot identify any evidence for a commensurate layer. However, that does not mean that the layers are not commensurate over longer distances or with an alignment that is not along the graphite principle directions.

The BPY molecule has been used extensively in scanning tunnelling microscopy (STM) measurements of complexation with other species on graphite. The STM image from pure 4,4'bipyridine has been reported (for example ${ }^{5}$, supplementary information S1b and ${ }^{7}$ supplementary Figure S1). However, no structural analysis was given and no unit cell proposed. The later of these STM papers proposes a schematic structure with the molecular axes essentially parallel to each other (with a small extent of orientational disorder) as schematically illustrated in Fugure 4(a). However, this molecular orientation gives a diffraction pattern that is significantly different to the experimental (Figure 4(b)). There are a number of differences between the diffraction approach used here and the STM measurements that may account for the differences in structure. For example, here pure BPY was adsorbed through the vapour phase. In the STM measurements the BPY is adsorbed from solution (toluene). 


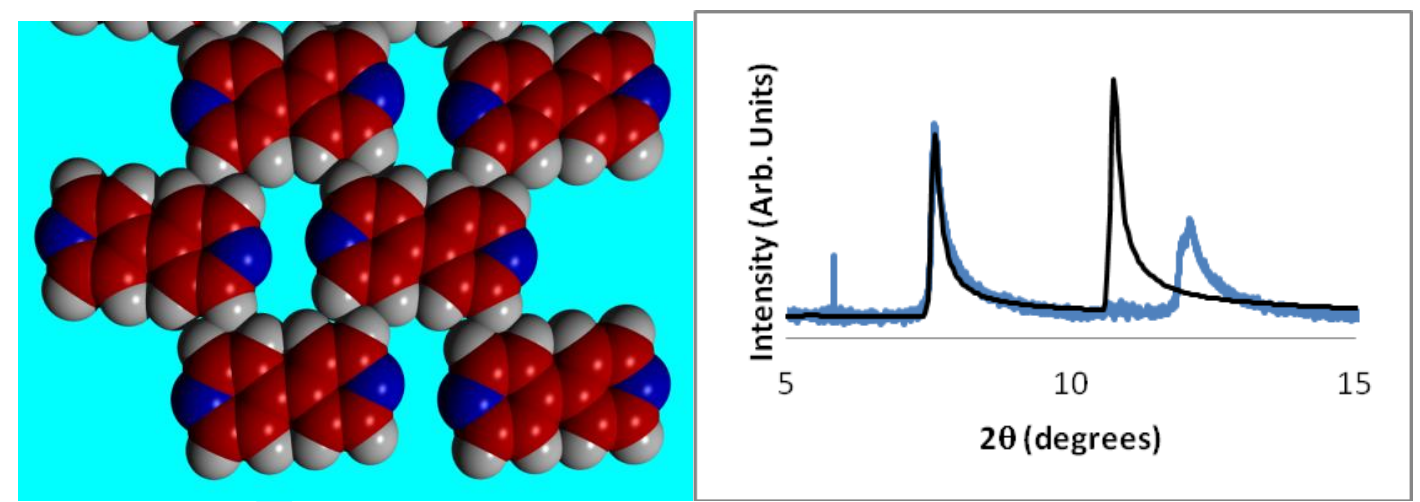

Figure 4. (a) Schematic illustration of the parallel arrangement of molecules in the monolayer structure of BPY proposed by STM. (b) Experimental and calculated patterns for the STM structure. (NB as no unit cell was given we have used the same unit cell as we have derived).

\section{Conclusions}

The formation of the solid monolayer of BPY has been observed by synchrotron Xray diffraction. The molecules from an essentially square lattice with plane group pgg andtwo molecules per unit cell which lay flat on the surface. The layer exhibits a small temperature dependence undergoing a change to a weakly rectangular unit cell at lower temperatures. Significantly the monolayers do not show any evidence of melting in the diffraction experiment at temperatures well above the bulk melting point, and even at these submonolayer coverages. Calorimetry data suggests the monolayer melting point is approximately $442 \mathrm{~K}$. This is a very unusual finding. The majority of physiorbed monolayers show a submonolayer melting point that is typically significantly lower (by a factor of 0.75 ) than the bulk. There are individual examples of other adsorbed molecules which exhibit a similar elevation in monolayer melting point above the bulk such as the alkyl amides ${ }^{1}$, but these represent the exception.

\section{Acknowledgements}

We thank the BP Institute, Diamond Light Source, and the University of Cambridge for financial support and facilities. Thanks to the DLS staff and scientists Chiu Tang and Stephen Thompson for beam time and technical support. This work was carried out with the support of the Diamond Light Source. The Herchel Smith find is acknowledged for a research fellowship (TF). Steven De Feyter is acknowledged for helpful discussions.

\section{References}

${ }^{1}$ T. Bhinde, S. M. Clarke, T. K. Phillips et al., Langmuir 26 (11), 8201 (2010).

${ }^{2}$ A. K. Bickerstaffe, N. P. Cheah, S. M. Clarke et al., J. Phys. Chem. B110, 5570 (2006). 
${ }^{3}$ K. Morishige and T. Kato, J. Chem. Phys. 111, 7095 (1999); K. Morishige and Y. Sakamoto, J. Chem. Phys. 103, 2354 (1995).

${ }^{4}$ R. Kuroda, E. Kishi, A. Yamano et al., J. Vac. Sci. and Technol. B9, 1180 (1991).

${ }^{5}$ S. Xu, S. Yin, and S. Xu, Applied Physics A 99, 99 (2010).

${ }^{6}$ S. De Fetyer and F. C. De Schryver, J. Phys Chem. B109, 4290 (2005).

${ }^{7}$ M. Li, Y. Yang, K. Zhao et al., J. Phys Chem. C 2008, 10141 (2008).

${ }^{8}$ M. Nishio, Y. Umezawa, K. Honda et al., Cryst. Eng. Comm 11, 1757 (2009).

${ }^{9}$ T. Steiner, Angew. Chem. Int. Ed. Engl. 41, 48 (2002).

${ }^{10}$ S. P. Thompson, J. E. Parker, J. Potter et al., Review of Scientific Instruments 80 (7), 075107 (2009)

${ }^{11}$ B E Warren, Phys. Rev. 59, 693 (1941).

${ }^{12}$ J K Kjems, L Passell, H Taub et al., Phys. Rev. B13, 1446 (1976).

${ }^{13}$ E B Sirota, H E King, H H Shao et al., J. Phys. Chem. 99, 798 (1995).

${ }^{14}$ N. M. Boag, K. M. Coward, A. C. Jones et al., Acta Cryst. C 55, 672 (1999); S. Kraft, E. Hanuschek, R. Beckhaus et al., Chem. Eur. J. 11, 969 (2005).

${ }^{15}$ A. Gavezzotti, J. Chem. Theory Comp. 1, 834 (2005).

${ }^{16}$ A. I. Kitaigorodskii, Organic chemical crystallography, edited by Editor. (Consultants Bureau, New York, 1961).

${ }^{17}$ S. M. Clarke and R. K. Thomas, Mol. Phys. 72, 413 (1991). 


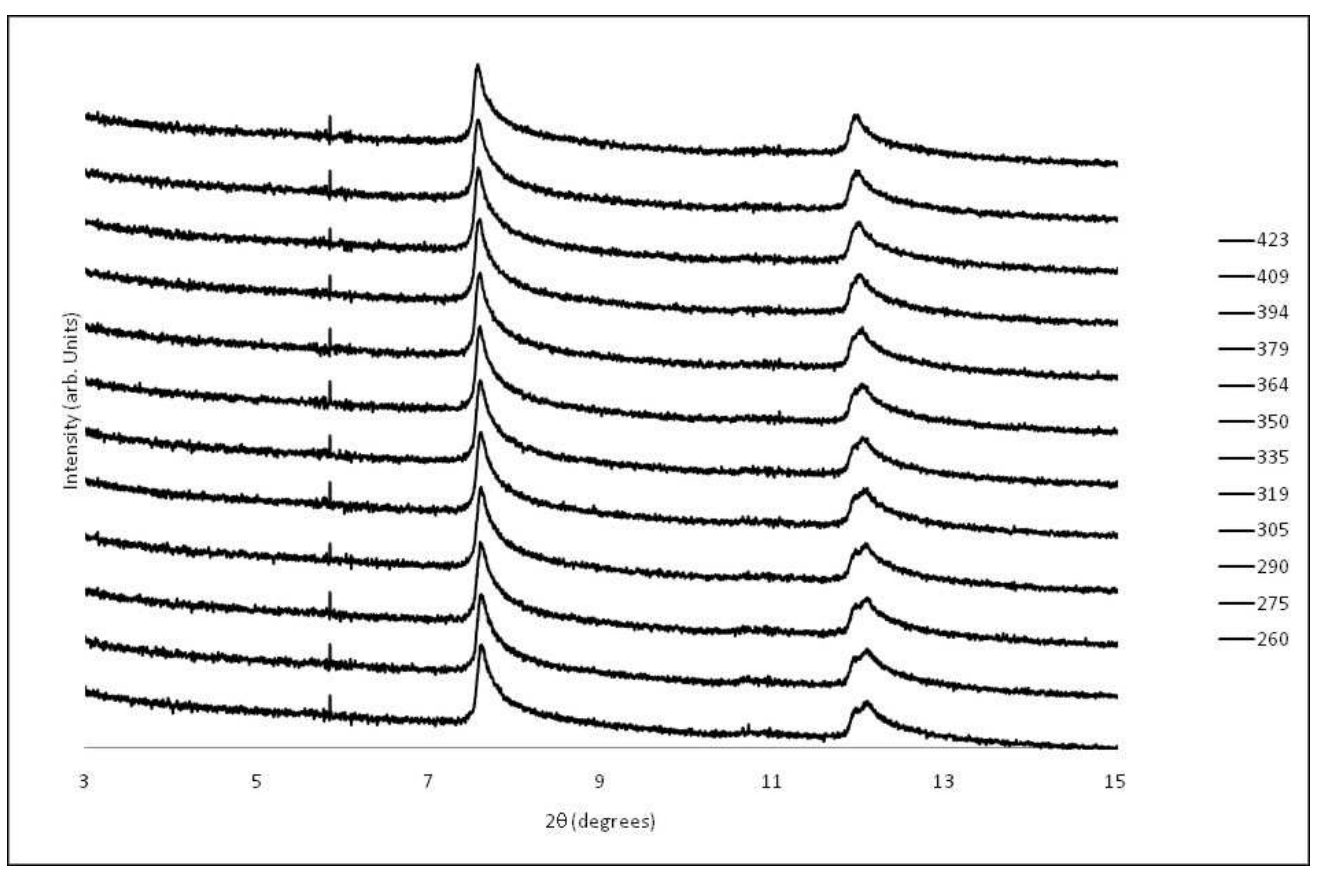

$80 \times 52 \mathrm{~mm}(300 \times 300$ DPI $)$ 